\title{
Review on the treatment of scars
}

\section{Daniel J. Callaghan}

Colorado Dermatology Specialists, Denver, CO 80237, USA.

Correspondence to: Dr. Daniel J. Callaghan, Colorado Dermatology Specialists, 3540 S Poplar St Suite 300, Denver, CO 80237, USA. E-mail: danieljcallaghan3@gmail.com

How to cite this article: Callaghan DJ. Review on the treatment of scars. Plast Aesthet Res 2020;7:66. http://dx.doi.org/10.20517/2347-9264.2020.166

Received: 16 Aug 2020 First Decision: 15 Sep 2020 Revised: 29 Sep 2020 Accepted: 26 Oct 2020 Published: 18 Nov 2020

Academic Editor: James E. Zins Copy Editor: Cai-Hong Wang Production Editor: Jing Yu

\begin{abstract}
Scarring is a major concern for patients. From acne scarring to surgical scars, scars can have a dramatically negative effect on one's self-esteem and are a common complaint for which patients seek treatment. This review will focus on the treatment of acne scarring including ice pick, boxcar and rolling scars, and also the treatment of surgical scars including atrophic and hypertrophic scars.
\end{abstract}

Keywords: Scarring, acne scarring, surgical scars

\section{INTRODUCTION}

Scarring is a condition that aesthetic physicians are frequently called upon to improve. The treatment of scars can be a rewarding albeit frustrating endeavor. Scars or scarring come in a number of varieties, and treatment must be tailored specifically for each patient. This chapter will focus on the treatment of acne scars and surgical scars as these are most routinely encountered in practice. Acne can produce ice pick, rolling or boxcar scars and treatment can vary widely from the use of fillers, trichloroacetic acid (TCA) or energy-based devices. Similarly, surgical scars can be treated with a number of modalities from injectables such as intralesional triamcinolone or 5-fluoruracil (5-FU) to resurfacing technologies.

\section{ACNE SCARS}

When it comes to facial rejuvenation, the treatment of acne scars is one of the things that can make the most dramatic improvement. While acne, and thereby acne scarring, generally occurs in one's teens or

cc) (i) The Author(s) 2020. Open Access This article is licensed under a Creative Commons Attribution 4.0 International License (https://creativecommons.org/licenses/by/4.0/), which permits unrestricted use, sharing, adaptation, distribution and reproduction in any medium or format, for any purpose, even commercially, as long as you give appropriate credit to the original author(s) and the source, provide a link to the Creative Commons license, and indicate if changes were made. 
twenties, patients can come in requesting treatment of acne scarring at any age. Acne scarring is generally classified as ice pick, rolling or boxcar, and the treatments of each subtype can vary. That said, patients typically have a variety of these subtypes at any given time, and this must be taken into account when deciding on the preferred treatment approach. One challenge in determining the optimal approach for treating acne scars is that there is a dearth of high-quality studies. The studies that exist are often small and underpowered, biased, without uniform baseline variables or outcomes or without long-term follow-up ${ }^{[1]}$.

\section{Ice pick scars}

Ice pick scars are deep but narrow $(<2 \mathrm{~mm})$ scars that look like they could have been created by an ice pick. Due to the depth of the scars, which can extend into the dermis, they are often more resistant to the typical treatment modalities used for rolling or boxcar scars. Although they have less treatment options in general, the ones that they have can provide superb results.

Punch excision is an excellent treatment option for ice pick scars. Although this is essentially trading a scar for a scar, the scars created by the punch excision itself often heal to the point they are difficult to see ${ }^{[2]}$. For the best cosmetic outcome, scars should be at least 4-5 $\mathrm{mm}$ apart to be treated at the same time. Otherwise, there will be too much tension on the skin surface for them to heal optimally. If scars are within 4-5 $\mathrm{mm}$ of one another, then waiting 4 weeks between treatments will provide the best long-term results ${ }^{[3]}$.

The use of TCA, particularly with the CROSS technique (chemical reconstruction of skin scars), has more recently emerged as a treatment option for ice pick scars. The CROSS technique involves using an instrument such as a syringe needle or a sharpened wooden applicator that is dipped into highconcentration TCA and then applied directly onto the scar. The desired endpoint is a white frosting of the scar. TCA creates coagulative necrosis of the epidermis, thereby increasing collagen production which ultimately results in improvement of the $\operatorname{scar}^{[2]}$.

In a study of 30 patients treated with the CROSS technique utilizing 100\% TCA every two weeks for a total of 4 sessions, Khunger et al. ${ }^{[4]}$ found that $73 \%$ of patients achieved excellent improvement in ice pick scars, whereas $20 \%$ achieved good improvement. Side effects of this technique include hypopigmentation which is largely transient, a burning or tingling sensation at the time of treatment and erythema or edema ${ }^{[4]}$. The CROSS technique has also been described using other chemicals such as $88 \%$ phenol with similar results as $\mathrm{TCA}^{[5]}$.

Although energy-based devices often provide less-than-satisfactory results for the treatment of ice pick scars, Ramesh et al.$^{[6]}$ found that ice pick scars responded better than rolling or boxcar scars to a fractional radiofrequency (FRF) device. Conversely, other studies found the opposite result ${ }^{[0,7]}$.

\section{Rolling scars}

Rolling scars are typically $\geq 4 \mathrm{~mm}$ in diameter and have soft, irregular walls which gives them a rolling appearance. These are caused by bands that tether the subcutis to the dermis. As such, the treatment of these scars generally targets these bands to improve their appearance.

Subcision has been a longstanding technique to target and release these bands. In this method, an instrument, such as a needle, is inserted into the subcutaneous plane and fanned back and further in an effort to sever these bands. Blunt blade subcision has also been used, in which a blunt blade is inserted in a single puncture site and is able to safely treat a wider area. A study by Barikbin et al.$^{[8]}$ involving 18 patients with mainly rolling scars found that this method led to marked improvement in 50\% of patients, while $33 \%$ had moderate improvement and $17 \%$ mild improvement ${ }^{[8]}$. 
Fillers have also been used to treat rolling scars. Sapra et al. ${ }^{[9]}$ looked into the use of poly-L-lactic acid for the treatment of rolling scars in 22 patients and found that $68.2 \%$ of patients had a satisfactory response as judged by blinded-evaluators. Hyaluronic acid and calcium hydroxyapatite have also been used to treat acne scars with success ${ }^{[10,11]}$.

Resurfacing is also used to treat rolling scars but will be discussed further below. Although it is not directly targeting the bands tethering the scars down, it can be effective in many circumstances.

\section{Boxcar scars}

Boxcar scars are wider than ice pick scars (1-4 mm in diameter) which gives them a U-shaped appearance. Their sharply demarcated edges are in contrast to the soft edges of rolling scars and can extend 0.1-0.5 mm into the dermis. Although boxcar scars are indeed a distinct form of acne scar, they are seldomly studied in isolation but rather are most often grouped together with the treatment of other types of scars.

In general, boxcar scars are treated with resurfacing, which can be performed with anything from a chemical peel or microneedling to a number of different energy-based devices. The aggressiveness of the treatment is often correlated to the results obtainable, but also must be weighed against the risks as well as the acceptable downtime for the patient.

Microneedling can be performed either with a dermaroller or a microneedling pen, and can be performed alone or with the use of a variety of topical applications to the pores created by microneedling such as platelet rich plasma (PRP). Alam et al.$^{[12]}$ performed a randomized, split-face study with a dermaroller on a number of morphologic acne scar types and found that after 3 treatments there was improvement in scarring, with a mean difference of 3.4 based on the quantitative global scarring grading system $(P=$ $0.03)^{[12]}$. A separate blinded, randomized controlled trial involving 42 patients comparing microneedling to a non-ablative fractional erbium 1,340-nm laser found that both were effective and that there was no statistically significant difference between the two $(P=0.264)$. Microneedling had fewer side effects and less downtime ${ }^{[13]}$. One study found microneedling combined with the use of PRP to be more effective than microneedling alone; however, an alternative study demonstrated no difference in these outcomes ${ }^{[14,15]}$.

Of the energy-based devices, fully ablative lasers typically offer the best cosmetic outcomes, but at the cost of the longest downtime and greatest risk for adverse events. Walia and Alster ${ }^{[16]}$ demonstrated a $75 \%$ improvement in atrophic acne scars at 18 months after high-energy $\mathrm{CO}_{2}$ laser treatment ${ }^{[16]}$. However, erythema lasting on average 3.5 months and a $36 \%$ incidence of hyperpigmentation help explain why this is not a commonly used modality to treat acne scars.

Fractional ablative lasers have helped to fill this void. They are effective but have a more acceptable recovery and side effect profile than fully ablative lasers. Bjørn et al. ${ }^{[17]}$ found that a fractional $\mathrm{CO}_{2}$ laser improved acne scarring with minor postoperative adverse effects, and that a treatment interval of either 1 month or 3 months did not influence the final outcome. Cho et al. ${ }^{[18]}$ compared the efficacy of fractional $\mathrm{CO}_{2}$ to nonablative fractional laser (NAFL) treatment with the 1,550-nm erbium:glass laser. They found that while the fractional $\mathrm{CO}_{2}$ laser demonstrated greater improvement, it was not statistically significant as there were only 8 patients in the study ${ }^{[18]}$. This improvement came at the cost of greater adverse effects including erythema and crusting.

NAFL are a mainstay in the treatment of acne scarring. With a lower downtime than ablative fractional lasers, patients often prefer them even if they may require more treatment sessions to achieve equal results. Sardana et al ${ }^{[19]}$ found that boxcar scars were most responsive to treatment with the 1,540-nm erbium:glass laser, demonstrating a $52.9 \%$ improvement compared to rolling scars which had a $43.1 \%$ improvement. 
Boxcar scars had a statistically significant improvement after four sessions $(P<0.05)$. Ice pick scars showed the lowest improvement rate of only $25.9 \%$, although this was not statistically significant $(P=0.09)^{[19]}$.

Radiofrequency devices can be monopolar, bipolar or fractional. Of these, FRF devices seem to provide the best results, with an expected improvement of $25 \%$ to $75 \%$ after 3 to 4 sessions. Although adverse effects are limited, the procedure itself can be associated with a significant amount of pain, even with nerve blocks or topical anesthesia ${ }^{[20,21]}$.

\section{Erythematous scars}

Beyond treating the textural changes of acne scarring, a typical complaint is post-inflammatory erythema. Although with time, this typically resolves on its own, but it can take months if not years. Vascular lasers such as the 595-nm pulsed dye laser (PDL) or 532-nm potassium titanyl phosphate (KTP) laser are widely used to treat this erythema because of their consistent and reliable results with minimal adverse effects.

\section{SURGICAL SCARS}

Physicians must be well-versed in the treatment of surgical scars. Every patient heals differently, and even the most precise surgical technique can lead to scarring. The treatment of an unfortunately placed or unsightly scar can be the most immediate thing a patient can do to improve his or her appearance, as the scar is often the first thing one's eye is attracted to upon seeing a person.

There are a number of things to consider when treating a surgical scar, including the timing of when interventions should be implemented, and what specific interventions should take place. Scars can manifest in a number of ways, and may be erythematous, raised or depressed.

Perhaps the earliest question that physicians or surgeons face in the management of scars is what should patients do in the immediate aftermath of surgery. Beyond appropriate wound care and timely suture removal, patients frequently inquire about the benefit of silicone gel sheeting. Although there have been a number of studies published touting the effects of silicone gel sheeting not only for preventing hypertrophic scars but also to treat those that are already present, a systematic review involving 20 trials and 873 patients found the evidence to be weak and heavily susceptible to bias ${ }^{[22]}$.

\section{Hypertrophic scars}

Hypertrophic scars are commonly treated with a number of modalities including intralesional kenalog (ILK), 5-FU or laser treatments. ILK has long been considered the first line treatment of hypertrophic scars. ILK suppresses inflammation, causes vasoconstriction which reduces the delivery of oxygen and nutrients to the scar and also has an antimitotic effect, inhibiting the growth of keratinocytes and fibroblasts. Additionally, it reduces plasma protease inhibitors which degrade collagen through collagenase ${ }^{[23]}$. The concentration used needs to be carefully considered for each individual scar and is dependent on the size and location of the scar. It is prudent to start with a lower dose with the expectation that multiple treatments may be necessary rather than risk using a higher dose which may lead to atrophy and pigmentary changes. It is much easier to treat conservatively than to have to treat additional complications down the line.

5-FU is a well-established albeit less commonly used technique for the treatment of hypertrophic scars. 5-FU is an antimetabolic agent that has been demonstrated to inhibit fibroblast proliferation and decrease collagen synthesis ${ }^{[24]}$. It can be used alone or in combination with ILK, and has been shown to decrease the risk of side effects of ILK when used in combination ${ }^{[25,26]}$. 5-FU should not be used in patients who have an infection, are pregnant or have anemia, leukopenia or bone marrow suppression. 5-FU is typically injected at a dose of $50 \mathrm{mg} / \mathrm{mL}$ for a maximum dose ranging from 50-150 mg. It can be diluted with ILK, which is typically diluted to a dose of 2 to $10 \mathrm{mg} / \mathrm{mL}$ dependent on the size and location of the scar. Care must be taken to avoid overtreating the scars, which can lead to atrophy, being more challenging to treat. 
Both ablative and non-ablative resurfacing are also popular techniques in treating hypertrophic scars. More recently, these have been combined with laser-assisted drug delivery with corticosteroids or 5-FU ${ }^{[27,28]}$. The use of 5-FU has been demonstrated to be as effective but with fewer side effects than the use of corticosteroids in laser-assisted drug delivery ${ }^{[28]}$.

Dermabrasion is one of the oldest methods used to revise scars. It can be done manually with sandpaper, or mechanically with a rotating wire brush or diamond fraise. Dermabrasion, particularly mechanical dermabrasion, is extremely operator dependent and carries a number of risks including making the scar worse. A randomized controlled trial comparing fractional ablative resurfacing to dermabrasion found that while both were effective, laser resurfacing was safer and showed quicker clinical recovery ${ }^{[29]}$. Conversely, in a randomized, blinded, split-scar study involving 14 patients, manual dermabrasion with sterilized sandpaper was demonstrated to be an effective but safe, simple and cost-effective treatment option for surgical scars ${ }^{[30]}$. Mechanical dermabrasion has fallen out of favor due to the risks associated with aerosolization of blood.

\section{Atrophic scars}

Atrophic surgical scars show a different set of challenges than hypertrophic scars and can generally be more difficult to treat.

Fractional laser therapy with either non-ablative or fully ablative lasers has been shown to improve the color, texture, thickness and patient satisfaction of atrophic surgical scars ${ }^{[31-33]}$. These lasers are effective because they stimulate neocollagenesis and dermal remodeling.

The use of fillers has been shown to improve the appearance of atrophic surgical scars. Both hyaluronic acid and calcium hydroxyapatite have been shown to be safe and effective with the additional benefit of having an immediate improvement ${ }^{[34]}$. One downside to the use of fillers is that the results are not permanent.

\section{Pigmentary changes}

Pigmentary changes can affect both hypertrophic and atrophic scars. The most common color change is typically erythema, resulting from the healing process that stimulates neovascularization. Scars can also be hyper- or hypopigmented. Although the previously mentioned techniques to treat scar texture may provide the added benefit of improving such pigmentary changes, in many cases this must be addressed separately.

Erythematous scars tend to be relatively receptive to treatment. PDL has long been used to treat erythematous surgical scars. It has been shown to be effective at both short and long pulse durations ${ }^{[35]}$. Although improvement in erythema should be the main objective when treating surgical scars with PDL, it has been shown to improve texture as well ${ }^{[36]}$. The 532-nm KTP laser is also well-established for the treatment of scars and has been demonstrated to be comparable in safety and efficacy to $\mathrm{PDL}^{[37]}$.

Hypopigmented scars can be challenging to treat; however, the combination of fractional resurfacing with the use of topical tretinoin, pimecrolimus or bimatoprost has been shown to be effective at re-pigmenting the $\operatorname{scar}^{[38]}$. Similarly, laser-assisted drug delivery of bimatroprost has been shown to be effective at repigmenting hypopigmented scars ${ }^{[39]}$.

\section{EMERGING TECHNOLOGIES}

Although this chapter focused on the most commonly used techniques to treat scars, the field of medicine is always working on emerging technologies that may one day complement or replace standard therapies. One such technology that may play a role in the management of scarring is laser speckle contrast imaging (LSCI). LSCI illuminates tissue with coherent laser light and then detects backscatter from the tissue 
which ultimately can be used to detect blood flow ${ }^{[40]}$. This is relevant to scarring because adequate tissue perfusion is necessary for the healing process to take place. This technology has been studied in patients with systemic sclerosis and can detect a reduction of blood perfusion in areas affected by Raynaud's phenomenon. It also has been used to demonstrate that a decrease in blood perfusion is found in patients with microangiopathy ${ }^{[4]}$. LSCI has also been used to help evaluate burn wounds which is important, because it can detect the severity of partial-thickness wounds, which in turn influences treatment ${ }^{[42]}$.

\section{CONCLUSION}

Scarring, regardless of etiology, is a challenging but treatable condition that can make a significant difference in the lives of patients. Although energy-based devices are the workhorses of many treatment regimens, they are not absolutely necessary, and any physician can be equipped to manage them. A thorough understanding of different types of scars is crucial to tailor a treatment course for individual patients. The treatment of acne scars differs depending on whether they are ice pick, rolling or boxcar scars. Surgical scars may be raised or depressed, or suffer from pigmentary changes, and treatments vary for each. As our understanding of the formation and maturation of scars continues to develop, new technologies will likely emerge to target scars or even inhibit their formation altogether.

\section{DECLARATIONS}

\section{Authors' contributions}

Contribute solely to the article: Callaghan DJ

\section{Availability of data and materials}

Not applicable.

\section{Financial support and sponsorship}

None.

\section{Conflicts of interest}

The author declared that there are no conflicts of interest.

\section{Ethical approval and consent to participate}

Not applicable.

\section{Consent for publication}

Not applicable.

\section{Copyright}

(c) The Author(s) 2020.

\section{REFERENCES}

1. Abdel Hay R, Shalaby K, Zaher H, et al. Interventions for acne scars. Cochrane Database Syst Rev 2016;4:CD011946.

2. Levy LL, Zeichner JA. Management of acne scarring, part II: a comparative review of non-laser-based, minimally invasive approaches. Am J Clin Dermatol 2012;13:331-40.

3. Jacob CI, Dover JS, Kaminer MS. Acne scarring: a classification system and review of treatment options. J Am Acad Dermatol 2001;45:109-17.

4. Khunger N, Bhardwaj D, Khunger M. Evaluation of CROSS technique with $100 \%$ TCA in the management of ice pick acne scars in darker skin types. J Cosmet Dermatol 2011;10:51-7.

5. Dalpizzol M, Weber MB, Mattiazzi AP, Manzoni AP. Comparative study of the use of trichloroacetic acid and phenolic acid in the treatment of atrophic-type acne scars. Dermatol Surg 2016;42:377-83.

6. Ramesh M, Gopal M, Kumar S, Talwar A. Novel technology in the treatment of acne scars: the matrix-tunable radiofrequency technology. 
J Cutan Aesthet Surg 2010;3:97-101

7. Peterson JD, Palm MD, Kiripolsky MG, Guiha IC, Goldman MP. Evaluation of the effect of fractional laser with radiofrequency and fractionated radiofrequency on the improvement of acne scars. Dermatol Surg 2011;37:1260-7.

8. Barikbin B, Akbari Z, Yousefi M, Dowlati Y. Blunt blade dubcision: an evolution in the treatment of atrophic acne scars. Dermatol Surg 2017;43 Suppl 1:S57-63.

9. Sapra S, Stewart JA, Mraud K, Schupp R. A canadian study of the use of poly-L-lactic acid dermal implant for the treatment of hill and valley acne scarring. Dermatol Surg 2015;41:587-94.

10. Goodman GJ, Van Den Broek A. The modified tower vertical filler technique for the treatment of post-acne scarring. Australas $J$ Dermatol 2016;57:19-23.

11. Goldberg DJ, Amin S, Hussain M. Acne scar correction using calcium hydroxylapatite in a carrier-based gel. J Cosmet Laser Ther 2006;8:134-6.

12. Alam M, Han S, Pongprutthipan M, et al. Efficacy of a needling device for the treatment of acne scars: a randomized clinical trial. JAMA Dermatol 2014;150:844-9.

13. Cachafeiro T, Escobar G, Maldonado G, Cestari T, Corleta O. Comparison of nonablative fractional erbium laser 1,340 $\mathrm{nm}$ and microneedling for the treatment of atrophic acne scars: a randomized clinical trial. Dermatol Surg 2016;42:232-41.

14. Asif M, Kanodia S, Singh K. Combined autologous platelet-rich plasma with microneedling verses microneedling with distilled water in the treatment of atrophic acne scars: a concurrent split-face study. J Cosmet Dermatol 2016;15:434-43.

15. Ibrahim MK, Ibrahim SM, Salem AM. Skin microneedling plus platelet-rich plasma versus skin microneedling alone in the treatment of atrophic post acne scars: a split face comparative study. J Dermatolog Treat 2018;29:281-6.

16. Walia S, Alster TS. Prolonged clinical and histologic effects from $\mathrm{CO} 2$ laser resurfacing of atrophic acne scars. Dermatol Surg 1999;25:926-30

17. Bjørn M, Stausbøl-Grøn B, Braae Olesen A, Hedelund L. Treatment of acne scars with fractional CO2 laser at 1-month versus 3-month intervals: an intra-individual randomized controlled trial. Lasers Surg Med 2014;46:89-93.

18. Cho SB, Lee SJ, Cho S, et al. Non-ablative 1550-nm erbium-glass and ablative 10600 -nm carbon dioxide fractional lasers for acne scars: a randomized split-face study with blinded response evaluation. J Eur Acad Dermatol Venereol 2010;24:921-5.

19. Sardana K, Manjhi M, Garg VK, Sagar V. Which type of atrophic acne scar (ice-pick, boxcar, or rolling) responds to nonablative fractional laser therapy? Dermatol Surg 2014;40:288-300.

20. Simmons BJ, Griffith RD, Falto-Aizpurua LA, Nouri K. Use of radiofrequency in cosmetic dermatology: focus on nonablative treatment of acne scars. Clin Cosmet Investig Dermatol 2014;7:335-9.

21. Boen M, Jacob C. A review and update of treatment options using the acne scar classification system. Dermatol Surg 2019;45:411-22.

22. O'Brien L, Jones DJ. Silicone gel sheeting for preventing and treating hypertrophic and keloid scars. Cochrane Database Syst Rev 2013;2013:CD003826.

23. Morelli Coppola M, Salzillo R, Segreto F, Persichetti P. Triamcinolone acetonide intralesional injection for the treatment of keloid scars: patient selection and perspectives. Clin Cosmet Investig Dermatol 2018;11:387-96.

24. Bulstrode NW, Mudera V, McGrouther DA, Grobbelaar AO, Cambrey AD. 5-fluorouracil selectively inhibits collagen synthesis. Plast Reconstr Surg 2005;116:209-21; discussion 222-3.

25. Darougheh A, Asilian A, Shariati F. Intralesional triamcinolone alone or in combination with 5-fluorouracil for the treatment of keloid and hypertrophic scars. Clin Exp Dermatol 2009;34:219-23.

26. Ren Y, Zhou X, Wei Z, Lin W, Fan B, Feng S. Efficacy and safety of triamcinolone acetonide alone and in combination with 5-fluorouracil for treating hypertrophic scars and keloids: a systematic review and meta-analysis. Int Wound J 2017;14:480-7.

27. Waibel JS, Wulkan AJ, Shumaker PR. Treatment of hypertrophic scars using laser and laser assisted corticosteroid delivery. Lasers Surg Med 2013;45:135-40.

28. Waibel JS, Wulkan AJ, Rudnick A, Daoud A. Treatment of hypertrophic scars using laser-assisted corticosteroid versus laser-assisted 5-fluorouracil delivery. Dermatol Surg 2019;45:423-30.

29. Christophel JJ, Elm C, Endrizzi BT, Hilger PA, Zelickson B. A randomized controlled trial of fractional laser therapy and dermabrasion for scar resurfacing. Dermatol Surg 2012;38:595-602.

30. Poulos E, Taylor C, Solish N. Effectiveness of dermasanding (manual dermabrasion) on the appearance of surgical scars: a prospective, randomized, blinded study. J Am Acad Dermatol 2003;48:897-900.

31. Cohen JL. Minimizing skin cancer surgical scars using ablative fractional Er: Yag laser treatment. J Drugs Dermatol 2013;12:1171-3.

32. Gokalp H. Evaluation of nonablative fractional laser treatment in scar reduction. Lasers Med Sci 2017;32:1629-35.

33. Tidwell WJ, Owen CE, Kulp-Shorten C, Maity A, McCall M, Brown TS. Fractionated Er: YAG laser versus fully ablative Er: YAG laser for scar revision: results of a split scar, double blinded, prospective trial. Lasers Surg Med 2016;48:837-43.

34. Kasper DA, Cohen JL, Saxena A, Morganroth GS. Fillers for postsurgical depressed scars after skin cancer reconstruction. $J$ Drugs Dermatol 2008;7:486-7.

35. Nouri K, Elsaie ML, Vejjabhinanta V, et al. Comparison of the effects of short- and long-pulse durations when using a 585-nm pulsed dye laser in the treatment of new surgical scars. Lasers Med Sci 2010;25:121-6.

36. Alster T, Williams C. Treatment of keloid sternotomy scars with $585 \mathrm{~nm}$ flashlamp-pumped pulsed-dye laser. Lancet 1995;345:1198-200.

37. Keaney TC, Tanzi E, Alster T. Comparison of $532 \mathrm{~nm}$ potassium titanyl phosphate laser and $595 \mathrm{~nm}$ pulsed dye laser in the treatment of erythematous surgical scars: a randomized, controlled, open-label study. Dermatol Surg 2016;42:70-6.

38. Massaki AB, Fabi SG, Fitzpatrick R. Repigmentation of hypopigmented scars using an erbium-doped 1,550-nm fractionated laser and 
topical bimatoprost. Dermatol Surg 2012;38:995-1001.

39. Waibel JS, Rudnick A, Arheart KL, Nagrani N, Gonzalez A, Gianatasio C. Re-pigmentation of hypopigmentation: fractional lasers vs laser-assisted delivery of bimatoprost vs epidermal melanocyte harvesting system. J Drugs Dermatol 2019;18:1090-6.

40. Heeman W, Steenbergen W, van Dam G, Boerma EC. Clinical applications of laser speckle contrast imaging: a review. J Biomed Opt 2019;24:1-11.

41. Ruaro B, Sulli A, Pizzorni C, Paolino S, Smith V, Cutolo M. Correlations between skin blood perfusion values and nailfold capillaroscopy scores in systemic sclerosis patients. Microvasc Res 2016;105:119-24.

42. Stewart CJ, Frank R, Forrester KR, Tulip J, Lindsay R, Bray RC. A comparison of two laser-based methods for determination of burn scar perfusion: laser Doppler versus laser speckle imaging. Burns 2005;31:744-52. 